\title{
Nonconverging Hysteresis Cycles in Random Spin Networks
}

\author{
O. Hovorka* and G. Friedman \\ Electrical and Computer Engineering Department, Drexel University, Philadelphia, Pennsylvania 19104, USA
}

(Received 23 March 2007; published 4 March 2008)

\begin{abstract}
Behavior of hysteretic trajectories for cyclical input is investigated as a function of the internal structure of a system modeled by the classical random network of binary spins. Different regimes of hysteretic behavior are discovered for different network connectivity and topology. Surprisingly, hysteretic trajectories which do not converge at all are observed. They are shown to be associated with the presence of specific topological elements in the network structure, particularly with the fully interconnected spin groups of size equal to or greater than 4 .
\end{abstract}

DOI: 10.1103/PhysRevLett.100.097201

PACS numbers: 75.60.Lr, 75.75.+a, 89.75.Fb

Although hysteresis is often illustrated by closed loops, there is no fundamental reason to expect that periodically varying external parameters should produce any kind of closed hysteretic trajectories in general. In fact, hysteretic trajectories which do not converge are observed experimentally in many systems. It is known, for example, that repeated mechanical loading and unloading of some materials leads to gradual accumulation of strain and eventual material failure, rather than stable hysteresis cycles [1]. Similar nonconvergent behavior in magnetic materials is manifested as a gradual shift of magnetization occurring under the action of periodic external field [2]. The question arises: What in the structure of a system determines the existence of nonconvergent hysteretic trajectories?

Here this issue is investigated by looking at binary spin networks as a way to model the structure of a system. Although it is most frequently employed to model magnetic materials, binary spin network is a classical prototype for studying hysteretic properties of many natural systems. Hysteretic cycles have been studied previously using the random field Ising model [3] and random networks of antiferromagnetically coupled spins [4], where the focus was mainly on the effects of disorder. Interesting memory effects have been discovered in spin-glass networks such as complementary point and reversal-field memories [5]. Particularly important property found to occur in ferromagnetic [6] and some antiferromagnetic networks [7] is the return point memory (RPM), which is responsible for the recovery of a microscopic state and formation of closed minor hysteresis loops after the very first external field cycle. Networks without RPM have been found to display only a gradual minor loop formation or subharmonic cycles [8] (cycles with a period multiple of the external field period, as illustrated in Fig. 1). Despite the substantial amount of work performed in this area, the ability of binary networks to describe nonconvergent hysteretic trajectories was not observed. The link between the network structure and the convergence character of hysteretic trajectories also remains largely unexplored.

In this Letter, we discover the emergence of nonconvergent trajectories with the appearance of certain topological structures. Specifically, we find that RPM observed at low network connectivity gives way to trajectories whose time of convergence to stable cycle grows without bound as the system size increases becoming nonconvergent in the thermodynamic limit.

As a prototypical system, we consider a classical ErdösRényi (ER) random network of antiferromagnetically coupled spins. It is a simple, yet convenient model of a complex network because its connectivity and topological structure can be tuned by adjusting a single parameterthe probability of connection between pairs of spins [9]. Generally, a random network of $N$ spins can be viewed as a random graph with $N$ nodes (vertices) connected by undirected edges representing pairwise interactions between the spins. Antiferromagnetic interactions between the spins

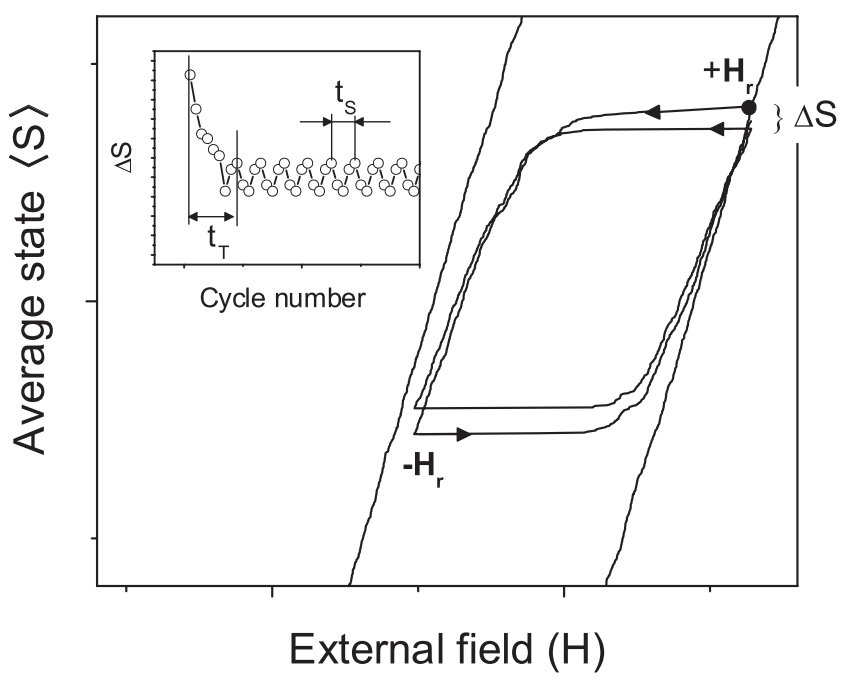

FIG. 1. Hysteretic cycles for two periods of external field. $S$ and $\langle S\rangle$ denote, respectively, the spin microstate and the average state (magnetization). $\Delta S$ is a measure of the cycle opening calculated by comparing spin patterns before and after each cycle. Inset: Dependence of $\Delta S$ on the external field period number. The steady state reached after initial transient time $t_{T}$ can contain simple minor loops with $t_{S}=1$ or subharmonic cycles with $t_{S}>1$. 
are here described by a matrix $J_{i j}=-J A_{i j}$, where $J$ is a positive number representing the interaction strength and $A_{i j}$ is an adjacency matrix of the graph with elements randomly chosen to be either 1 or 0 .

Dynamics of the system is governed by minimization of the standard Ising-type energy:

$$
G=-\frac{1}{2} \sum_{i j} J_{i j} s_{i} s_{j}-H \sum_{i} s_{i}+\sum_{i} f_{i}\left(s_{i}\right)
$$

where $s_{i}$ denotes the \pm 1 spin variable and $H$ is the external field. The first and the second sum in (1) are, respectively, the spin-spin and the spin-external field interaction energies. Additionally, there is a symmetric double-well potential energy $f_{i}$, due to which any isolated spin $s_{i}$ flips from -1 to +1 at the field-threshold $+\alpha_{i}$, while the flipping from +1 to -1 occurs at the field $-\alpha_{i}$. The positive threshold magnitudes $\alpha_{i}$ will be viewed here as random variables and mimic a structural disorder in the system. Note, that the difference between the "up" and "down" thresholds, $+\alpha_{i}>-\alpha_{i}$, results in an inherent hysteretic behavior of each spin. Such classical hysteretic spins are frequently used in phenomenological models of hysteresis as representations of, e.g., single-domain magnetic grains, capillary pores in absorbing materials, decision making agents in socioeconomic systems, etc. [10].

The local field $h_{i}$ governing the flipping of each spin is obtained as a variation of (1) with respect to $s_{i}$ and equals

$$
h_{i}=-\delta G / \delta s_{i}=-J \sum_{j} A_{i j} s_{j}+H .
$$

We consider standard field driven adiabatic dynamics [6] where, at each time step, the spin states are updated only if $h_{i} s_{i}<-\alpha_{i}$. For a network with negative interactions such dynamics may produce avalanches with backward flipping spins when $\Delta H \Delta s_{i}<0$, resulting in a nonmonotonic variation of state of the entire system even when the field $H$ varies monotonically [11]. Here we avoid such backward spin flips by requiring that $J \max \left(d_{i}\right)<\min \left(\alpha_{i}\right)=$ $\alpha_{\min }$ is satisfied for the particular network, where $d_{i}=$ $\sum_{j} A_{i j}$ is a degree of vertex $i$ in the network, i.e., the coordination number of the spin $s_{i}$. This results in a simple avalanche behavior consisting of single spin flips, for which $\Delta H \Delta s_{i}>0$, and in monotonic state variation. We also note that our implementation selects randomly which spin to flip should more than one spin become unstable after the field increment. However, such bifurcations in the system's dynamics are not observed in reality because every threshold $\alpha_{i}$ in the system is different due to the quenched-in disorder. In simulations, we use Gaussian distribution of $\alpha_{i}$ 's with variance $\sigma$ and mean $\mu \gg \sigma$ [12]. Such an assumption is natural for various realistic systems such as, e.g., magnetic films with very strong perpendicular anisotropy or patterned nanostructures [13].
It turns out that even the single spin-flip dynamics introduced above yields complex minor loop behavior. This is demonstrated here by simulating hysteretic trajectories corresponding to periodic cycles of the external field (illustrated in Fig. 1) obtained by increasing the field starting from the negative saturation to the point $H_{r}$ with the average spin state (magnetization) $\langle S\rangle$, then decreasing the field to $-H_{r}$ and returning back to $H_{r}$. The ability of the network to recover its state when the field returns to $H_{r}$ will be quantified by the cycle opening, $\Delta S$, equal to percent difference in a number of spins which did not return to the original state.

We observe that the cycle opening $\Delta S$ depends on the degree $d$ (i.e., connectivity) of the network, which is defined as an average over all $d_{i}$ in the particular network realization. For low connectivity $d \ll 1$, the system returns to the same state at the end of the very first cycle. This is expected since the majority of spins is isolated or form couples and such antiferromagnetic systems are known to have a RPM [14]. As $d$ increases the cycle opening $\Delta S$ becomes nonzero. However, a well-pronounced increase of $\Delta S$ is observed only after the percolation threshold of the network at $d \sim 1$, when a giant spin cluster appears [9]. Then, after reaching a maximum, $\Delta S$ starts to decrease to zero as $d$ approaches the network size $N$ with the limit $\Delta S=0$ obtained for fully connected network $d=N$. Such behavior is expected since the fully connected spin network can be viewed as a mean-field Preisach model, which has been shown to have RPM [15].

Numerical tests for different network sizes $N$ (up to $50^{2}$ ), different disorder $\sigma$ and different average energy per spin $\Delta=\left\langle J \sum_{j} A_{i j} s_{j}\right\rangle \approx J d\langle S\rangle$, revealed that the cycle opening $\Delta S$ depends on the ratio $\Delta / \sigma$, rather than on $\Delta$ and $\sigma$ separately. In addition, $\Delta S$ was observed to be independent of the network size as long as $d \ll N$, and we found no dependence on the mean $\mu$ in the assumed limit $\mu \gg \sigma$. Since the probability of finding various topological interconnection structures in the ER network, such as trees and cliques (completely interconnected subgraphs) [9], depends both on $d$ and $N$, these results demonstrate that the first cycle opening does not depend on the topological properties of the network.

It turns out, however, that the network topology does determine the minor loop formation behavior. To show this we studied the dependence of $\Delta S$ on the number of external field cycles, the network size $N$, the $\Delta / \sigma$ ratio, and the connectivity parameter $d$. An example of the $\Delta S(\Delta / \sigma, d)$ function is given by the contour plots in Figs. 2(a)-2(d) for four subsequent field cycle numbers and a fixed network size $N=50^{2}$. The figure illustrates that in the low $(\Delta / \sigma$, d)-parameter region closed minor loops with $\Delta S=0$ appear already after a few initial field cycles [Figs. 2(a) and 2(b)]. Similar behavior, termed as "tilting effect" (bascule), is frequently observed in magnetism especially for clean ferromagnets [2]. It is often attributed to the coupling 

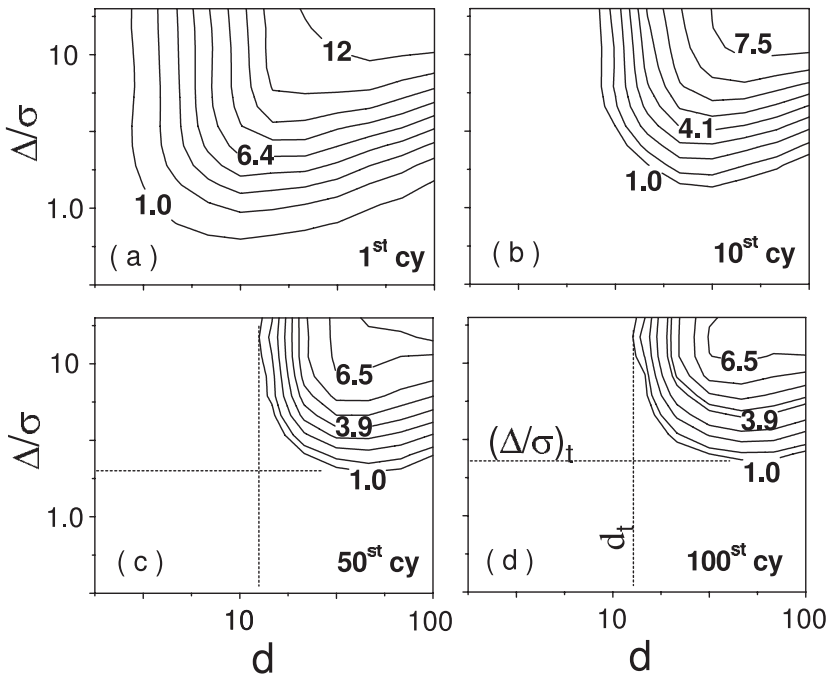

FIG. 2. (a)-(d) Contour maps showing the cycle opening $\Delta S$ for different values of interaction energy $\Delta / \sigma$ and the network degree $d$ (note the logarithmic scale of $\Delta / \sigma$ and $d$ axes) for, respectively: $1 \mathrm{st}, 10 \mathrm{th}, 50 \mathrm{th}$, and 100th cycle. The lower bounds for the "limiting" region with nonconverging loops in (d) correspond to $(\Delta / \sigma)_{t}=2.3$ and $d_{t} \sim 13$. All minor loops have been obtained by reversing the field at $H_{r}$ where $\langle S\rangle=0.2$, when the effect was most pronounced. Data are for $N=50^{2}, \sigma=0.1$ and averaged over 50 random graph and disorder realizations. Error bars level is about $1 \%$.

between only a small number of magnetic domains (consistently with the low connectivity network picture considered here).

For the parameter region bounded from below by certain critical values $(\Delta / \sigma)_{t}$ and $d_{t}$ [Fig. 2(d)] the behavior changes dramatically and closed minor cycles often do not form even after 100 field periods. Quite surprisingly, we find that the value $d_{t}$ is remarkably close to the theoretical value $N^{1 / 3}$ at which the ER network is known to undergo a topological transition associated with the emergence of cliques of size 4 (fully interconnected groups of 4 spins) [9]. This behavior then persists for a large range of higher network connectivities $d$, and qualitatively does not change even when cliques of size 5 and greater emerge (note that 5-cliques appear at about $d \sim N^{1 / 2}$ [9], i.e., at $d \sim 4 d_{t}$ for a system size studied in Fig. 2).

To be more specific, two new types of cyclic behaviors are observed: cycles with a very long transient time, $t_{T}>$ 100 , and subharmonic cycles with a spin pattern recovering repeatedly with period $t_{S}>1\left(t_{T}\right.$ and $t_{S}$ are defined in the Fig. 1). Note that subharmonic cycles were also observed previously for some spin-glass networks [8]. Here, the period $t_{S}$ is found to grow with the number of 4-cliques present in the network. However, this growth is substantially slower than the increase of the network size $N$ and no conclusions can be made regarding behavior of the subharmonic cycle length in the thermodynamic limit $(N \rightarrow$ $\infty)$. On the other hand, the transient length $t_{T}$ grows rapidly, as shown in Fig. 3(a) by the log-log dependence of $\left\langle t_{T}\right\rangle$ on the average number of 4-cliques $\left\langle C_{4}\right\rangle$ (averaged over 100 network realizations). Dependence on the network size is plotted in Fig. 3(b) and follows the exponential law $\left\langle t_{T}\right\rangle \sim \exp (N / \tau)$. The parameter $\tau$ has been obtained by fitting and according to the inset in Fig. 3(b) $\tau \rightarrow \infty$ in the limit $\left\langle C_{4}\right\rangle \rightarrow 0$. This means that, for $d>d_{t}$, when cliques of size 4 or greater exist, the $\left\langle t_{T}\right\rangle$ grows faster than $N$, implying that hysteretic trajectories will not converge in the thermodynamic limit $(N \rightarrow \infty)$.

Why would cliques matter? Based on the observations for particular network realizations, the following intuitive explanation can be given. During any field cycle we observe that groups of spins flipping up and down are usually different. They differ not so much in their size, but mainly in their position within the network. For a chain of spins, for example, a group of spins flipping up and down often shifts its position after one cycle as if propagating along the chain. A very similar situation occurs for treelike or for single isolated loop network structures, where groups of flipping spins propagate along simple paths during the field cycling. In such networks, hysteretic cycles effectively stabilize when the propagating groups of flipping spins encounter obstacles in the form of spins whose thresholds are too large to be switched during the cycle. The situation changes qualitatively for networks with cliques. In this case more than a single loop can be often drawn through a pair of spins. As a result, groups of flipping spins can spread out, split, or go around other spins whose thresholds are too large to flip during cycles. This leads to complex and seemingly random variations in the pattern of flipping
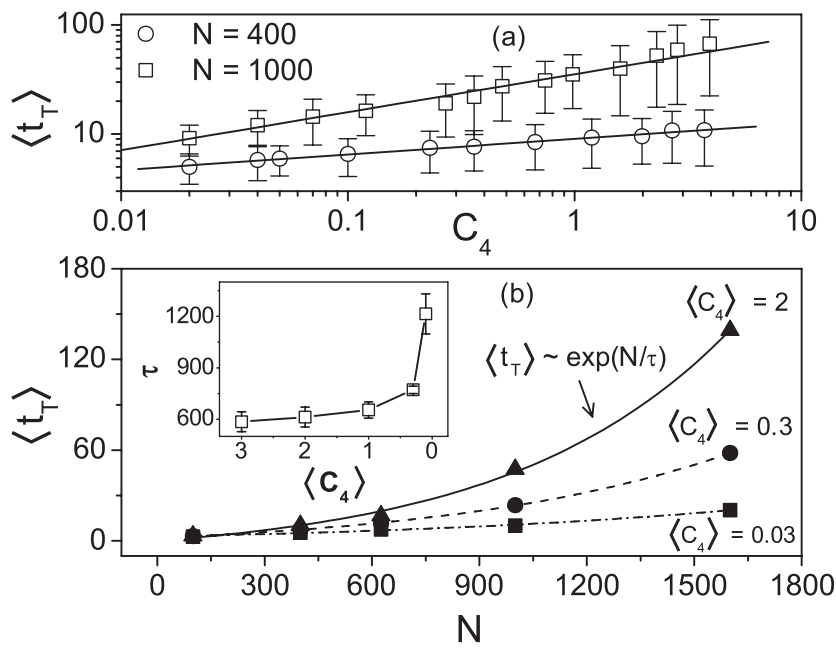

FIG. 3. (a) Dependence of the transient length on the number of 4-cliques for $N=20^{2}$ and $10^{3} .\left\langle t_{T}\right\rangle$ and $\left\langle C_{4}\right\rangle$ are averages over 100 network realizations. (b) $\left\langle t_{T}\right\rangle$ as a function of $N$ obtained for $\left\langle C_{4}\right\rangle=0.03,0.3$ and 2 . Lines for different $\left\langle C_{4}\right\rangle$ are exponential fits. Data correspond to reversal magnetization $\langle S\rangle=0.2$, $\Delta / \sigma=10$, and $\sigma=0.1$. Inset: dependence of the exponential fit parameter $\tau$ on $\left\langle C_{4}\right\rangle$. $\tau$ grows without bound with diminishing number $C_{4}$ of 4 -cliques. 
spins as cycling continues and to the nonconvergence of hysteretic trajectories. However, a more detailed quantitative investigation is required to fully support this explanation [16].

In conclusion, the present antiferromagnetic binary spin network model displays several qualitatively very different regimes, including a regime where hysteretic trajectories do not converge. Assuming the single spin-flip field driven dynamics, the determining factors appear to be the ratio between the interaction strength and the disorder variance, the network connectivity, and its topology.

We thank Professors J. P. Sethna and H. Katzgraber for useful comments and suggestions. This work is supported in part by NSF Grant No. ECS-0304453.

*Present address: CIC nanoGUNE Consolider, Mikeletegi Pasealekua 56, E-20009 Donostia-San Sebastián, Spain.

[1] T. Broom and R. K. Ham, Proc. R. Soc. A 242, 166 (1957).

[2] L. Néel, J. Phys. Radium 20, 215 (1959); P. J. Thompson and R. Street, J. Phys. D 29, 2789 (1996); L. P. Lévy, J. Phys. I (France) 3, 533 (1993); V. Hajko et al., IEEE Trans. Magn. 10, 128 (1974).

[3] J.P. Sethna, K. A. Dahmen, and Ch. R. Myers, Nature (London) 410, 242 (2001).

[4] K. Malarz et al., Physica (Amsterdam) 373A, 785 (2007); B. Tadić, K. Malarz, and K. Kułakowski, Phys. Rev. Lett. 94, 137204 (2005).

[5] H. G. Katzgraber et al., Phys. Rev. Lett. 89, 257202 (2002); H. G. Katzgraber and G. T. Zimanyi, Phys. Rev. B 74, 020405(R) (2006); M.S. Pierce et al., Phys. Rev. Lett. 94, 017202 (2005).
[6] J. P. Sethna, K. Dahmen, S. Kartha, J. A. Krumhansl, B. W. Roberts, and J. D. Shore, Phys. Rev. Lett. 70, 3347 (1993).

[7] J. M. Deutsch, A. Dhar, and O. Narayan, Phys. Rev. Lett. 92, 227203 (2004).

[8] E. Vives and A. Planes, Phys. Rev. B 50, 3839 (1994); J. M. Deutsch and O. Narayan, Phys. Rev. Lett. 91, 200601 (2003); J. M. Deutsch, T. Mai , and O. Narayan, Phys. Rev. E 71, 026120 (2005).

[9] S. N. Dorogovtsev and J.F.F. Mendes, Evolution of Networks (Oxford University Press, New York, 2003); R. Albert and A.-L. Barabási, Rev. Mod. Phys. 74, 47 (2002). Erdös-Rényi random graphs: the node degree $d_{i}$ (coordination number) is a random variable following binomial distribution. For a large number of vertices $N$ in the graph, the connection probability $p$ and the average degree $d=\left\langle d_{i}\right\rangle$ are related as $d \sim p N$. As $p$ (or $d=p N$ for fixed $N$ ) increases, at first only loops and then also cliques appear in the treelike network existing initially at low $p$.

[10] The Science of Hysteresis, edited by G. Bertotti and I. D. Mayergoyz (Academic Press, New York, 2006).

[11] P. Shukla, Physica (Amsterdam) 233A, 242 (1996); P. Shukla, R. Roy, and E. Ray, Physica (Amsterdam) 276A, 365 (2000); 275A, 380 (2000).

[12] Strictly speaking, due to the truncation of the random numbers below $\alpha_{\min }$, the threshold distribution slightly differs from the Gaussian. However, the probability for observing a random number below $\alpha_{\min }=\mu-3 \sigma$ is negligible.

[13] P. Moriarty, Rep. Prog. Phys. 64, 297 (2001); J. I. Martin et al., J. Magn. Magn. Mater. 256, 449 (2003).

[14] L. Néel, C. R. Acad. Sci., Paris 246, 2313 (1958); 246, 2963 (1958).

[15] M. Brokate and E. Della Torre, IEEE Trans. Magn. 27, 3811 (1991).

[16] G. Friedman and O. Hovorka (unpublished). 